\title{
UN ESTUDIO ACÚSTICO-CONTRASTIVO DE LAS VOCALES DEL PORTUGUÉS BRASILEÑO USADO COMO HERRAMIENTA EN EL PROCESO DE ENSEÑANZA DEL PORTUGUÉS COMO LENGUA EXTRANJERA
}

\author{
Rosa Elena García \\ Universidad de Los Andes \\ Mérida-Venezuela \\ garciarosa77@hotmail.com
}

\section{RESUMEN}

En esta investigación, se realizó un estudio contrastivo para comparar acústicamente las vocales orales y nasales del portugués brasileño producidas por hablantes nativos y aprendices de portugués como lengua extranjera. Esto con la finalidad de determinar las diferencias entre la configuración de los formantes (F1, F2) de ambos grupos. Fue posible identificar las vocales en frases portadoras, las cuales fueron grabadas y procesadas con el software Praat. Con los valores de los formantes (F1 y F2) de las vocales fueron elaborados sendos triángulos acústicos que comparados mostraron los siguientes resultados: Con relación a las vocales orales producidas por los aprendices: (1) todas las vocales son más altas y posteriores que las producidas por los hablantes nativos, (2) las vocales / $\varepsilon$ e / se encuentran mucho más próximas; sin embargo, la vocal $/ \varepsilon /$ producida por los aprendices es más posterior y más alta que la vocal /e/ de los hablantes nativos, (3) como ocurre con las vocales $/ \varepsilon, e /$, las vocales /oo/ se encuentran en la misma zona de articulación; no obstante, la vocal / o / de los aprendices tiende a ser más anterior y más baja que la vocal /o/ producida por hablantes nativos, (4) la vocal /u/ tiende a ser más baja que la producida por los hablantes nativos, (5) la vocal /e/ de los aprendices se hace frontal casi la /i/.

\section{ABSTRACT}

In this research, a contrastive study was realized to compare acoustic the oral and nasal vowels of the Brazilian Portuguese produced by native speakers and apprentices of Portuguese as foreign language. This with the purpose of determining the differences between the configuration of the formants (F1, F2) of both groups. It was possible to identify the vowels in carrying phrases, which were recorded and tried by the software Praat. With the values of the formants (F1 and F2) of the vowels there were elaborated triangles acoustic that compared showed the following results: In relation to the oral vowels produced by the apprentices: (1) All the vowels are high and later than the produced ones for the native speakers, (2) The vowels $/ \varepsilon$ e/ are much more near; nevertheless, the vowel $/ \varepsilon /$, produced by the apprentices is more later and higher than the vowel /e/ of the native speakers, (3) since it happens with the vowels $/ \varepsilon, \mathrm{e} /$ the vowels /o o / are in the same zone of joint; nevertheless, the vowel $/ \mathrm{s} /$ produced by the apprentices tends to be more previous and lower than the vowel /o/ produced by native speakers, (4) the vowel $/ \mathrm{u} /$ tends to be lower than the produced one for the native speakers, (5) the vowel /e/ produced by the apprentices makes to them, it is frontal almost /i/. In relation to the nasal vowels 
Con respecto a las vocales nasales /ã ẽ ĩ õ ũ / producida por los aprendices, el estudio reveló que: (1) todas son más altas que las producidas por hablantes nativos, (2) las vocales / ẽ i / se ubican en una posición posterior, (3) la vocal / ũ / se retrae, (4) Las vocales nasales /ã õ ũ/se mantienen en posición posterior.

PALABRAS CLAVE: Análisis acústico, enseñanza de segundas lenguas y enfoque comunicativo. /ã ẽ ĩ õ ũ / produced by the apprentices, the study revealed: (1) They all are high than the produced ones for native speakers, (2) the vowels / e $\tilde{i} /$ are located in a later position, (3) the vowel / ũ / is re-brought, (4) The nasal vowels /ã õ ũ/ are kept in later position.

KEY WORDS: acoustic analysis, teaching of the second languages and communicative approach.

\section{Introducción}

Las dificultades en la pronunciación de los fonemas vocálicos del portugués brasileño por parte de aprendices venezolanos de portugués como lengua extranjera fue lo que motivó esta investigación, que se inició con la siguiente hipótesis: Con respecto a su cualidad acústica, hay diferencias notables entre las vocales del portugués brasileño producidas por hablantes nativos y las vocales del portugués brasileño producidas por aprendices de portugués como lengua extranjera. Para demostrar la hipótesis, se realizó un análisis acústico-contrastivo porque se considera que un estudio de este tipo servirá a los profesores como un instrumento en el que pueden visualizar, en este caso, las diferencias en la pronunciación de las vocales orales y nasales del portugués brasileño producidas por aprendices con relación a la producción de los mismos fonemas por parte de hablantes nativos. Asimismo, se considera que un estudio acústico-contrastivo ofrecería pistas sobre dónde están las dificultades en la producción de los fonemas vocálicos por parte de los aprendices. Todo lo anterior, con el propósito de ayudar a los aprendices a transitar el camino hacia la competencia comunicativa con mayor confianza y desenvoltura.

No obstante, la descripción de cualquier fenómeno debe poseer un fundamento teórico que respalde sus principios. Es por esta razón que, a seguir, se describen algunos de los conceptos relacionados con el proceso enseñanza-aprendizaje de segundas lenguas o lenguas extranjeras. En primer lugar, se comienza por la comunicación desde una perspectiva psicolingüística, los enunciados que hacen posible la comunicación, la comunicación en el contexto enseñanza-aprendizaje de lenguas extranjeras o segundas lenguas, el enfoque comunicativo, la competencia comunicativa en la lengua materna, la competencia comunicativa en el contexto enseñanza-aprendizaje de lenguas extranjeras o segundas lenguas y la actuación. En segundo lugar, se definen nociones como transferencia, predicción de la transferencia, transferencia 
entre lenguas genealógicamente próximas y el Portuñol. Para finalizar, se presenta el sistema vocálico del portugués brasileño.

\section{La comunicación desde una perspectiva psicolingüística}

Desde el punto de vista psicolingüístico, según Dubois (1973), la comunicación es el conjunto de fases sucesivas en cuyo transcurso la significación que un interlocutor asocia a los sonidos es semejante a la que el oyente asocia a esos mismos sonidos. Por otro lado, los elementos que constituyen cada mensaje están enlazados necesariamente con el código a través de una correspondencia interna y con el mensaje por una correspondencia externa. Así, siguiendo a Jakobson (1973), puede afirmarse que el lenguaje, en sus diferentes formas, utiliza ambos modos de relación. Del mismo modo, para que la comunicación sea efectiva debe existir una conexión entre los símbolos usados por el emisor y los que el receptor conoce e interpreta. Sin tal conexión, el mensaje es inútil y no consigue ningún fin.

En este sentido, el acto de la comunicación verbal, en una primera instancia, es codificación y decodificación de los elementos constitutivos de todo mensaje oral; es decir, se le concede a la comunicación una función primaria distintiva. De acuerdo con Jakobson (1973), tanto el hablante como el oyente disponen del mismo "fichero de representaciones prefabricadas": el emisor del mensaje verbal elige una de estas "posibilidades preconcebidas". Por parte del receptor, se supone una elección equivalente a partir del mismo conjunto de "posibilidades ya previstas y preparadas". Así, la acción de comunicar y comunicarse requiere para ser eficaz que aquellos que intervienen en el evento utilicen un código común.

\section{Los enunciados que hacen posible la comunicación}

Desde este punto de vista, la comunicación se hace efectiva cuando locutor y receptor conocen las reglas que regulan la lengua o idioma. Sólo así, el oyente podrá interpretar los enunciados escogidos por el hablante en un momento determinado. De allí que se haga necesario indagar sobre los enunciados que permiten la comunicación.

La comunicación oral se realiza por medio de enunciados. Sin embargo, no existe una teoría propiamente dicha que defina el enunciado a partir de criterios lingüísticos precisos, se asimila el enunciado a la frase o a un conjunto de frases que se suceden. La caracterización del enunciado está ligada al concepto mismo de frase: representa un segmento más o menos extenso de la cadena hablada en la transmisión de los datos de la experiencia (Martinet, 1972). 
Jakobson (1973) asume que el receptor se apropia del enunciado o mensaje a través de una combinación de partes constitutivas (frases, palabras, fonemas, etc.) escogidas de entre el repertorio de todas las partes constitutivas posibles. En este sentido, Martinet (1972) ha propuesto que los enunciados que permiten comunicar determinadas experiencias se organizan en dos planos. En un primer plano, o primera articulación, los enunciados se enlazan linealmente en unidades mayores o unidades significativas dotadas de sentido: oraciones, sintagmas, palabras, etc. En esta primera articulación, se encuentran las unidades que se conocen como monemas o morfemas. Estas unidades tienen entre sus objetivos indicar la función gramatical de las palabras dentro de los enunciados. En el plano de la segunda articulación, cada monema se articula, a su vez, en su significante por medio de unidades carentes de sentido y distintivas que se conocen con el nombre de fonemas.

\section{La comunicación en el contexto enseñanza-aprendizaje de lenguas extranjeras o segundas lenguas}

Como puede observarse, se comienza describiendo la comunicación como un proceso psicolingüístico. Sin embargo, esta investigación está enmarcada en el proceso enseñanza-aprendizaje del portugués como lengua extranjera, por lo que se hace indispensable aludir al concepto de comunicación en este ámbito. Se ha señalado que la lengua es, en primer lugar, un conjunto de relaciones internas. Pero, la lengua no es solamente un sistema compuesto de la suma de diferentes formas, estructuras, y, en definitiva, vocablos variados, sino básicamente un medio para la comunicación. De allí que, en el contexto enseñanza-aprendizaje de lenguas extranjeras, el término comunicación presenta una connotación más extensa.

Para Pérez Martín (1995, citado por Cabrera, 2001:43-44):

La comunicación es una forma de interacción social, altamente imperceptible, al tiempo que creativa; que impone restricciones en cuanto a lo que es apropiado o no (en lo que a uso se refiere); que se lleva a cabo bajo circunstancias psicológicas que condicionan la actuación del usuario (limitaciones de memoria, cansancio, distracciones,...) que implica un usuario auténtico de la lengua y que se juzgará como efectiva (o no) en función de los resultados concretos alcanzados.

\section{Enfoque comunicativo}

Como consecuencia de este punto de vista, se considera que el rasgo predominante en la enseñanza de una LE es el de ofrecer a los aprendices una 
práctica que los conduzca directamente a intercambios comunicativos de diversas formas o lo que se conoce como enfoque comunicativo en la enseñanza de lenguas extranjeras. Para Ortega (2000), el objetivo principal en la adquisición de una LE es el desarrollo de la capacidad para producir y comprender mensajes con la intención de interactuar con los demás. En este sentido, Cabrera (2001: 44) ha indicado lo siguiente:

Lo que realmente interesa en el aula de LE es la actuación directa del alumno por medio de la lengua meta, la persecución de diversas finalidades comunicativas, al tiempo que se les presta atención a las relaciones entre las diversas oraciones que componen su discurso, los contextos y las situaciones en que aparecen, pues todos los parámetros determinarán el entorno más inmediato de dicho encuentro comunicativo, a la luz del cual se juzgará si los enunciados articulados por los alumnos (esto es, la producción en LE) son, o no, correctos y adecuados.

Así pues, el enfoque comunicativo representa una contribución, un aporte decisivo en el camino hacia la lengua meta. Haciendo parte de este enfoque, se encuentra la competencia comunicativa.

\section{La competencia comunicativa en lengua materna}

El término competencia comunicativa en el ámbito del aprendizaje lenguas fue desarrollado por Hymes (1972) en los años setenta. Para Hymes, la competencia comunicativa es el término más general para la capacidad comunicativa de una persona, esta capacidad engloba tanto el conocimiento de la lengua como la habilidad para utilizarla. En palabras de Hymes, la competencia comunicativa se relaciona con saber cuándo hablar, cuándo no, de qué hablar, con quién, cuándo y dónde y en qué forma; es decir, se trata de la capacidad que posee el hablante para construir enunciados que no sólo sean gramaticalmente correctos sino también socialmente apropiados. Podemos decir, siguiendo a Hymes, que la adquisición de tal competencia está enmarcada por la experiencia social, las necesidades, las motivaciones, y, principalmente, la acción que representa una fuente que motiva la necesidad de expresar y expresarse con relación a la experiencia.

La competencia comunicativa descrita por Hymes nos habla de la existencia de un hablante oyente-real, dotado de ciertas posturas sociales y miembro de una determinada comunidad lingüística. Así, la competencia comunicativa exige no sólo las habilidades para activar una lengua, sino saber situarse en el contexto comunicativo de cada comunidad específica. Esta perspectiva, nos sitúa, entonces, en el dominio semántico de la lengua cuya función básica es comunicar. Nos interesa la lengua en funcionamiento, comunicando nuestras experiencias, integrando grupos sociales e incorporando la noción de referente. 


\section{La competencia comunicativa en el ámbito enseñanza aprendizaje de lenguas extranjeras o segundas lenguas}

Posteriormente, en la didáctica de segundas lenguas, Savignon (1971) utilizó la expresión competencia comunicativa para referirse a la capacidad que faculta a los aprendices para comunicarse con sus compañeros de clase en la lengua meta; distinguió así esta capacidad (que permite un uso significativo de la lengua que se desean aprender) de la capacidad que les permite repetir los diálogos propuestos en los textos. Más adelante, Canale (1983) describe la competencia comunicativa como un conjunto de cuatro competencias que subyacen en la competencia comunicativa. Las dos primeras subcategorías definen el sistema en sí mismo, las dos últimas tienen relación con aspectos de la comunicación. Las subcategorías son: 1) La competencia gramatical o lingüística que representa el conocimiento morfológico, sintáctico, fonológico y semántico de la lengua. Esta competencia está asociada al dominio de la lengua como sistema de signos organizados. 2) La competencia sociolingüística hace referencia a la capacidad del hablante para producir mensajes en un determinado contexto, teniendo en consideración un conjunto de factores: las normas de cortesía, los distintos registros, estatus de los participantes, actitudes y elementos cinéticos. 3) La competencia textual que está asociada al conocimiento necesario para producir y comprender enunciados (coherentes y cohesivos) que superen el nivel oracional; así como también la capacidad de interpretarlos y 4) La competencia estratégica que está sujeta al conocimiento y a la habilidad del aprendiz para utilizar estrategias de comunicación verbal con la finalidad de compensar las deficiencias en la comunicación debido a algunas fallas de las competencias anteriormente descritas.

A estas cuatro competencias, Van Ek (1986) añade la competencia sociocultural y la competencia social. La competencia sociocultural es la capacidad de una persona para utilizar una determinada lengua relacionando la actividad lingüística comunicativa con unas determinadas estructuras cognitivas. En otras palabras, se trata de que los aprendices no solo dominen el código lingüístico de la LE, sino que sean capaces de actuar de forma comunicativamente adecuada. La competencia social hace referencia a las habilidades y estrategias socio-cognitivas con las que el hablante cuenta en la interacción social. Dentro de las competencias sociales se incluyen el autocontrol, la autorregulación emocional, el reforzamiento social y las habilidades para la resolución de problemas.

Es importante señalar que Valdman (1975) hace una distinción fundamental entre competencia lingüistica y competencia comunicativa. La competencia lingüística es el sistema abstracto de reglas que enlaza los hechos lin- 
güísticos observables con la significación, mientras que la competencia comunicativa es el sistema abstracto de reglas que asocia los actos de habla observables (dar órdenes, hacer preguntas, persuadir, entretener, etc.) a las estrategias comunicativas. Dicho de otra manera, la competencia comunicativa se refiere a la adquisición de reglas de uso de la lengua y debe intuirse de la observación de actos de habla.

\section{La actuación en una segunda lengua o una lengua extranjera}

Al hablar de competencia comunicativa no puede dejar de mencionarse la actuación. La actuación se define como el uso real de la competencia por medio de expresiones en circunstancias efectivas de comunicación. El aprendiz utiliza, para lograr la comunicación, parte del conocimiento que posee de su lengua materna, al tiempo que va afianzando el conocimiento de la lengua extranjera debido, precisamente, a la práctica significativa del proceso. El objetivo central que mueve a los individuos a intentar el aprendizaje de cualquier lengua es alcanzar la capacidad que les permita hacer uso de la misma como instrumento de comunicación.

En este punto, es importante señalar que esta investigación destaca el componente fonético-fonológico de la competencia lingüística que subyace en la competencia comunicativa, por considerar que el desconocimiento de este componente conduce a las interferencias del sistema lingüístico materno con la lengua meta. Las interferencias son uno de los mayores problemas, tanto para profesores como para aprendices, puesto que impiden la adquisición del nuevo sistema y se transforman en trabas, que si no son tratadas a tiempo, se convierten en fallas irremediables. Las trabas ocurren, entre muchas otras razones, por el desconocimiento del componente fonéticofonológico de la lengua que se quiere aprender, o la tendencia natural de los aprendices a transferir el propio sistema lingüístico a la lengua meta.

Con relación a los problemas que ocasiona el desconocimiento del componente fonológico de la lengua extranjera, Trubetzkoy (1973: 46) ya había señalado que:

Las personas se apropian del sistema de su lengua materna, y cuando oyen hablar otra lengua emplean involuntariamente, para el análisis de lo que oyen, la "criba" fonológica que le es habitual, es decir, la de su lengua materna. Pero como esta "criba" no se adapta a la lengua extranjera, surgen numerosos errores e incomprensiones. Los sonidos de la lengua extranjera reciben una interpretación fonológica inexacta debido a que se los ha hecho pasar por la "criba" fonológica de su propia lengua. 
Acerca del componente fonético-fonológico, Cagliari (1978) dice que el alumno debe tener conciencia de cómo oye los sonidos y cómo debe articularlos. Esto nos lleva a discutir ahora cómo el alumno toma conciencia de esas realidades. Esa conciencia activa del alumno, en términos de pronunciación, se adquiere a través de una conciencia fonética de los mecanismos percepción y de habla, así como del conocimiento práctico de los sonidos de la lengua y de su valor fonológico. En otras palabras, debe alertarse al alumno sobre las realidades fonético-fonológicas de la lengua que desea aprender.

El profesor de lenguas extranjeras debe incorporar elementos de fonología señalando los valores distintivos que los sonidos tienen en determinadas lenguas. La noción de oposición entre fonemas debe ser introducida desde el inicio o señalada, siempre que sea necesario, hasta que el alumno cree una conciencia fonológica. "La conciencia fonológica se adquiere a través de mecanismos de percepción y producción de los sonidos de la lengua meta" (Cagliari, 1978: 4). Por esta razón, en el proceso enseñanza-aprendizaje de lenguas extranjeras, el profesor debe preocuparse fundamentalmente por producir todos los sonidos de forma correcta. Puesto que, es él, en principio, el único que puede ayudar a los aprendices a crear la conciencia fonológica que les permitirá decodificar los significantes y, por ende, apropiarse de los significados que esos significantes refieren. Todos los demás factores lingüísticos y de comunicación verbal son esencialmente dependientes de una emisión hablada correctamente.

Con relación a la pronunciación, Llisterri (2003) advierte que es una capacidad que todo alumno necesita dominar cuando aprende una lengua extranjera; es por esto que debería hacer parte de los contenidos de cualquier diseño curricular y el profesor tendría que incorporarla a las actividades de clase, de la misma forma que introduce las dirigidas a la práctica de la expresión escrita o de la comprensión oral.

Así pues, pieza fundamental en el aprendizaje de una segunda lengua es el reconocimiento de cómo se pronuncian y estructuran los mensajes que posibilitan la comunicación por lo que un estudio de las unidades menores (fonemas) sería de gran importancia. Siguiendo a Cagliari (1978), puede decirse que existen implicaciones basadas en hechos ligados a la mecánica del habla, que conocemos y pueden ayudar a racionalizar el trabajo por etapas debidamente planeadas, controlando la producción y la calidad, de tal modo, que a partir de un cierto punto del programa se pueda prever, de cierto modo, que el alumno sabe la lengua, bajo ciertos aspectos. Asimismo, Cagliari señala que saber una lengua no significa que el alumno la usará automáticamente, pero ciertamente tendrá mucha facilidad para esto. Por otro lado, afirma 
que quien no sabe la lengua tendrá el mínimo de tranquilidad lingüística para usarla, y en la medida en que su intranquilidad aumente, mayores serán las dificultades que tendrá en hablar la lengua extranjera (que realmente no sabe). Cagliari añade que no es raro encontrar personas que conocen la estructura sintáctica de la lengua extranjera, conocen el sentido de las palabras y, a pesar de eso, no consiguen hablar la lengua porque no dominan una pronunciación razonable de esa lengua. Esas mismas personas, opina el autor, tienen, normalmente, gran dificultad en entender lo que otras personas dicen, a pesar de conocer todas las palabras que le fueron dirigidas o entender lo que le fue dicho, cuando escrito.

En este mismo sentido, según Corder (1992: 247), parte del aprendizaje de una L2 "consiste en descubrir los agrupamientos en conjuntos, de las series de los sonidos; es decir, aprender los fonemas o unidades funcionales de sonidos de la lengua meta".

\section{La transferencia}

El término transferencia fue descrito por Lado (1973) como la tendencia que posee el aprendiz para trasladar la estructura gramatical de la lengua materna a la lengua que desea aprender. El estudiante tiende a transferir las formas de las oraciones, los recursos de modificación, el número, el género, y los tipos de modificación de su lengua nativa. Esa tendencia representa la fuente principal de dificultad o facilidad de aprender la estructura de la lengua meta: aquellas estructuras que sean parecidas serán fáciles de aprender porque podrán transferirse y funcionar satisfactoriamente en la lengua extranjera. Aquellas estructuras que sean diferentes serán difíciles porque cuando se las traslada no funcionan idóneamente en el idioma extranjero.

No obstante, según Corder (1992), en esta afirmación de Lado, coexiste la implicación de que los términos dificultad y diferencia son sinónimos. Esta implicación no es evidente. Con relación a los términos dificultad y diferencia, Nickel (1971, citado por Corder, 1992: 226) apunta lo siguiente, "El que un rasgo determinado de la lengua meta sea diferente de la lengua materna, no necesariamente implica que sea difícil de aprender". Corder (1992: 227) delimitó muy bien esta idea:

En efecto, existen pruebas de que algo totalmente "nuevo" o diferente puede resultar más fácil de dominar que algo que es sólo ligeramente diferente; por ejemplo, cuando un sonido muy semejante existe en las dos lenguas, pero en diferentes circunstancias fonéticas, puede haber un problema de aprendizaje mayor que en el caso de un sonido totalmente nuevo. En este momento, parece aconsejable simplemente hacer notar que la dificultad es, sin duda, una cuestión psicolingüistica, mientras que la diferencia es lingüística. 
Según Llisterri (2003), se produce un paralelismo entre unidades iguales (similitud estructural entre la L1 y la L2) que producirán la transferencia positiva. Mientras que, unidades diferentes (cuando en la L2 no existen elementos que se parezcan a la L1) que producirán la transferencia negativa. Para Hammerly (1982), es importante destacar que Lado (1973) describió la transferencia como una tendencia, no como un proceso que involucra una predicción perfecta. Al mismo tiempo, Gass (1996) considera que Lado (1973) asumía la transferencia como la imposición de información de la lengua nativa, en forma de reflejos directos, sobre las expresiones u oraciones de la L2.

Por su parte, Kellerman (1979, 1983, citado por Gass, 1996: 325) distinguió los términos transferencia e interferencia de la siguiente forma: "La interferencia es el resultado lingüístico del influjo de una lengua sobre otra, mientras que la transferencia es el mecanismo o proceso psicológico que antecede a la interferencia, en la que está incluida la toma de decisiones del aprendiz con relación a los elementos lingüísticos transferibles". Obviamente, esto ocurre, sin dejar de lado la dimensión de similitud o disimilitud de las lenguas en el proceso de toma de conciencia por parte del aprendiz de aquellos elementos que son transferibles.

Otros autores han abordado este tema desde distintos ángulos. Entre ellos se encuentran Zobl (1980-1982, citado por Gass, 1996); Schachter (1983-1992, citada por Gass, 1996) y Kellerman (1977, citado por Gass, 1996). Un hallazgo significativo en estas investigaciones es el hecho de que la influencia de la lengua materna no ocurre sólo como destellos lingüísticos directos, sino que éstos reflejan principios fundamentales de la organización de la lengua. Como resultado de los trabajos de estos investigadores se asume que en el proceso de adquisición de una L2, la transferencia involucra el uso de información lingüística de la lengua materna o, también, de otras lenguas conocidas por el aprendiz. Zobl supone que las transferencias y las influencias evolutivas no son procesos opuestos, sino procesos que interactúan. El efecto de tal interacción puede manifestarse en: a) una prolongación o retardo en la reestructuración de una regla de la lengua, $b$ ) en el número de reglas que interfieren en el camino desde la adquisición de una forma a otra. De este modo, la Ll introduce variación hacia una sucesión progresiva en el proceso de aprendizaje de una L2.

Los estudios realizados por Schachter (1983, 1992, citada por Gass, 1996) han definido la transferencia en términos más amplios, incorporando todo el conocimiento lingüístico previo, incluyendo el conocimiento que se considera "imperfecto" que un aprendiz pueda tener de una determinada lengua. Gass (1996:320) interpreta la apreciación de Schachter (1983-1992) de la siguiente manera: 
Supongamos que un aprendiz construye una hipótesis acerca de una determinada estructura de la L2, una hipótesis que no se parezca a su lengua nativa ni a la lengua que desea aprender; esta hipótesis irá a influenciar las futuras hipótesis que el aprendiz pueda ir construyendo. No podría decirse que en la formación de esa hipótesis hay una influencia de la lengua nativa, porque la influencia no proviene de la lengua nativa. De manera similar, podría decirse que la influencia proviene del conocimiento lingüístico previo $y$, en consecuencia, podría incorporarse ese tipo de efecto a un modelo general de influencia lingüística en el aprendizaje de una lengua extranjera.

Para Gass (1996: 321), una visión más amplia de este tipo de influencia tiene en cuenta fenómenos como: a) reglas estructurales limitadas, b) transferencia de organización tipológica, c) diferentes caminos de adquisición, d) fallas de uso (neutralización), e) sobreproducción de ciertos elementos, f) atención adicional dada a la lengua meta, resultando en mayor rapidez para aprender $y, g)$ efectos diferenciales de forma de prestigio social. Estos elementos eran difícilmente detectables dentro de las concepciones anteriores de transferencia.

\section{Predicción de la transferencia}

Para predecir la transferencia, según Kellerman (1977, citado por Gass, 1996), se requiere cierta habilidad para descubrir dónde ésta podría producirse o no. De manera que una posible respuesta ampliaría la pregunta: ¿Qué es lo que se transfiere? Antes de responder a esta pregunta, sería conveniente definir los términos transferencia e interferencia. Brown (2000) ha definido la transferencia como un término general que describe el alcance de la actuación previa o conocimiento previo en el aprendizaje de una L2. Al mismo tiempo, señala, a partir de este fenómeno, dos conceptos: transferencia positiva y transferencia negativa. La transferencia positiva ocurre cuando el conocimiento previo beneficia las tareas del aprendizaje; es decir, cuando un ítem anterior se usa para obtener resultados correctos en la L2. Mientras que la transferencia negativa ocurre cuando la actuación rompe el desarrollo de una tarea. Este último término puede también ser llamado interferencia. En este caso, el conocimiento previo interfiere con el material subsiguiente; es decir, un ítem anterior se transfiere asociado con un ítem a ser aprendido incorrectamente o incorrectamente asociado con otro ítem a ser aprendido.

En la propuesta de Kellerman (1979, 1983, citado por Gass, 1996), hay dos factores relevantes que interactúan en la determinación de los elementos transferibles. Por una parte, se encuentra la percepción del aprendiz en relación a la distancia entre L1 y L2; en segundo lugar, el rasgo marcado de una estructura dada de la L1. Existen elementos de una lengua que los hablantes nativos consideran irregulares, no frecuentes o semánticamente "opacos". En términos de 
Kellerman, estos elementos son altamente marcados y menos transferibles que las formas regulares o frecuentes (elementos transferibles). El primer factor está relacionado con los elementos específicos, aquellos que el aprendiz considera únicos en su lengua. Los aspectos neutrales son los que el aprendiz cree que son comunes en su lengua nativa. Estos factores deben ser considerados cuando se refiere al proceso enseñanza-aprendizaje de dos lenguas genealógicamente próximas como el español y el portugués. Principalmente, porque, según Ferreira (1977a), los niveles de transferencia e interferencia son mayores.

\section{Transferencia entre lenguas genealógicamente próximas}

Debido a la gran semejanza entre dos lenguas cercanas, tales como el español y el portugués, la enseñanza del portugués, para hablantes de español, posee características particulares. Por un lado, es cierto que, en el proceso enseñanza-aprendizaje del portugués para hispanohablantes, existe un mayor margen de comprensión en los primeros contactos, por otro lado, la semejanza entre las dos lenguas impide que, muy avanzado el proceso de aprendizaje, el hablante de español se comunique en portugués sin las constantes interferencias de su lengua nativa. Santos (1998) afirma que, generalmente, se observa en los hablantes de español que aprenden portugués el uso del español con algunas pinceladas de portugués. En este sentido, Ferreira (1977a) expone que la relación portugués-español lleva a los aprendices a considerar la semejanza entre las lenguas como un elemento favorable a la adquisición. Y es, según Ferreira (1977a), aquí donde se percibe la primera gran contradicción porque se evidencia el desconocimiento de la distancia entre la L1 y L2 por parte de los aprendices hispanohablantes. Esta situación conduce al surgimiento de una lengua intermedia que se conoce como Portuñol.

\section{El portuñol}

Buarque (1999) ha definido el Portuñol como el resultado de la mezcla de los códigos lingüísticos portugués y español. Así, el Portuñol es la expresión más inmediata entre las dos lenguas y, se asume como una etapa de interlengua en el proceso enseñanza-aprendizaje de portugués para hablantes de español o de español para hablantes de portugués. Selinker (1972) propuso el nombre de interlengua para definir los sistemas intermedios entre la lengua materna y la lengua meta. Los sistemas poseen características propias, siendo influenciados, no sólo por la interferencia de la lengua materna, sino también por otros factores entre los cuales puede incluirse la propia interlengua en formación. Según Selinker (1972), sólo un 5\% de los aprendices de 
una segunda lengua logran alcanzar la perfecta competencia en la lengua meta; todos los demás desarrollan una interlengua en la que la incidencia de rasgos lingüísticos fosilizados es condición permanente.

\section{La fosilización}

Selinker (1972) define la fosilización como rasgos y subsistemas lingüísticos que los hablantes de una lengua nativa particular conservarán en su interlengua (relativa a una lengua meta particular) sin importar la edad de los aprendices o la cantidad de información e instrucción que ellos reciban en la lengua meta. Según Selinker, existe una estructura psicológica latente, genéticamente determinada, activada cada vez que el aprendiz quiere expresar una idea en la lengua meta. Esta estructura posee cinco procesos centrales: 1) transferencia de la lengua materna, 2) transferencia de entrenamiento, 3) estrategias de aprendizaje de la L2, 4) estrategias de comunicación en la L2 y, 5) sobregeneralización de los aspectos lingüísticos de la lengua meta, responsables por el surgimiento de la fosilización en el aprendizaje de la interlengua. Sin embargo, para Santos (1998), la fosilización es la interrupción del desarrollo sistemático de la interlengua, asociado a uno de los cinco procesos, propuestos por Selinker (1972). Schumann (1976, citado por Santos, 1998) expone que la fosilización de una interlengua sucede cuando el proceso de integración cultural del aprendiz en la sociedad meta se interrumpe; ésta puede ser superada si existe una necesidad real de integración a la sociedad meta, por parte del aprendiz. Con relación a los aprendices hispanos que aprenden portugués como segunda lengua, Santos (1998) afirma que la hipótesis de Schumann (1976) no se explicaría exactamente ya que, además de las semejanzas lingüísticas, existe un gran paralelismo en los aspectos culturales, lo que permite una rápida integración cultural si se compara con otros extranjeros que van al Brasil a aprender portugués. Al respecto, Santos indica que, en el caso del hablante de español, se cree que la fosilización sucede al inicio del proceso del desarrollo de la interlengua, debido a la gran facilidad de contacto de este grupo con los hablantes nativos. Esto se explicaría por la gran semejanza entre las dos lenguas, lo que origina una cierta "adaptación" por parte del aprendiz. Santos agrega que esto hace que pronto se detenga el avance hacia la lengua meta. El bloqueo en la comunicación es casi inexistente puesto que la interlengua (Portuñol) facilita el entendimiento. Para Santos, la fosilización, en el contexto enseñanza-aprendizaje del portugués para hispanohablantes, ocurre, fundamentalmente, por tres motivos: 1) el entendimiento recíproco que se establece entre los hablantes de las dos lenguas, 2) el ritmo de las clases, lento para los hablantes de español, por lo tanto tedioso, 3) textos didácticos elaborados para extranjeros de cualquier nacionalidad. 
En la opinión de Ferreira (1995b), en el aprendizaje de otras lenguas que no son genealógicamente tan próximas, se acepta la interlengua de los aprendices como un fenómeno lingüístico natural: un proceso progresivo del alumno en dirección a la lengua meta. Pero, tratándose de hablantes de español aprendiendo portugués, se observan algunos aspectos que pueden ser contradictorios. El hecho de ser lenguas hermanas, mutuamente inteligibles, y que pueden ser consideradas como dialectos una de la otra, tiene, en cierta forma, ventajas. Entre ellas: a) el hablante de español no se considera principiante; b) el grado de comprensión permite al hablante de español tener más seguridad y mayor capacidad de "riesgo", lo que representa un factor favorable para que exista un mayor avance en la dirección de la nueva lengua; c) la identificación entre las culturas es, también, un factor muy importante. Visto así, según Ferreira, la primera tendencia sería considerar la semejanza como positiva; sin embargo, existe una contradicción: si por un lado, la semejanza facilita el entendimiento; por otro, no deja que el aprendiz intente comunicarse sin las constantes interferencias de su lengua materna. La proximidad lleva a los aprendices a percibir más las semejanzas que las diferencias; esto hace que hablen una mezcla de español y portugués (Portuñol); sin embargo los aprendices "piensan que realmente están hablando la lengua meta. A partir de esta creencia, resulta muy difícil enseñar una lengua a quien cree que ya la habla" (Ferreira, 1995b: 143). También, sugiere Ferreira que el aprendizaje del portugués para hispanohablantes lleva implícito la creencia de la aparente facilidad, lo que trae como consecuencia que los aprendices no lo tomen muy en serio en el inicio.

\section{Sistema vocálico del portugués}

Con la intención de precisar el ámbito en el que se inscribe este trabajo, se realizó la distribución de los sonidos vocálicos del portugués, siguiendo a Cristófaro (2001). Primero, las vocales orales y posteriormente, las vocales nasales.

\begin{tabular}{|c|c|c|c|}
\cline { 2 - 4 } \multicolumn{1}{c|}{} & Anteriores & Central & Posteriores \\
\cline { 2 - 4 } \multicolumn{1}{c|}{} & No labializadas & No labializada & Labializada \\
\cline { 2 - 4 } \multicolumn{1}{c|}{} & Agudas & Neutra & Graves \\
\hline Cerradas & /i/ & & $/ \mathrm{u} /$ \\
\hline Semicerradas & $/ \mathrm{e} /$ & & $/ \mathrm{o} /$ \\
\hline Semiabierta & $/ \mathrm{e} /$ & & $\mathrm{J} /$ \\
\hline Abierta & & $/ \mathrm{a} /$ & \\
\hline
\end{tabular}

Cuadro 1. Subsistema de vocales orales del portugués brasileño según Cristófaro (2001) 
Como puede observarse en el Cuadro 1 , las vocales orales del portugués brasileño, desde el punto de vista acústico, son siete: tres agudas /i e $\varepsilon$ /, tres graves /u o $/$ / y una neutra /a /. Dependiendo de la abertura de la cavidad, las vocales se dividen en: dos vocales cerradas /i u/ dos semicerradas /e o/ dos semiabiertas /ع / y una abierta /a /.

Las vocales nasales del portugués brasileño, según Cristófaro (2001), se clasifican del siguiente modo:

\begin{tabular}{|c|c|c|c|}
\cline { 2 - 4 } \multicolumn{1}{c|}{} & Anteriores & Central & Posteriores \\
\cline { 2 - 4 } \multicolumn{1}{c|}{} & No labializadas & No labializada & Labializada \\
\hline & Agudas & Neutra & Graves \\
\hline Cerradas & $/ \tilde{i} /$ & & $/ \tilde{u} /$ \\
\hline Semicerradas & $/ \tilde{e} /$ & & $/ \tilde{o} /$ \\
\hline Abierta & & $/ \tilde{a} /$ & \\
& & & \\
\hline
\end{tabular}

Cuadro 2. Subsistema de las vocales nasales del portugués brasileño según Cristófaro (2001)

El cuadro 2 muestra la distribución de las vocales nasales del portugués brasileño desde el punto de vista acústico: dos agudas / ĩ ẽ /, dos graves / ũ õ / y una neutra / ã /. Con relación a la abertura, hay dos cerradas / ĩ ũ/, dos semicerradas / ẽ o / y una abierta / ã /. Comparado con el sistema oral, el subsistema nasal presenta una disminución en el número de vocales, de siete vocales orales pasa a cinco vocales nasales: / i è ẽ ã ũ õ /. En estos fonemas el rasgo de nasalidad es distintivo. No obstante, como sucede en español, todos los fonemas del subsistema oral pueden ser nasalizados por asimilación a una consonante nasal.

Desde el punto de vista fisiológico, se puede realizar la representación de las distintas vocales por medio de los triángulos articulatorios que intentan dar una idea, lo más exacta posible, de la situación articulatoria de cada vocal en la cavidad bucal. Desde el punto de vista acústico, también es factible la representación de un sistema vocálico por medio de los triángulos acústicos (Quilis, 1988).

En este sentido, se plantearon objetivos que sirvieron como pautas durante el proceso de recolección y procesamiento de los datos.

\section{Objetivos de la investigación}

(1) Realizar una categorización acústica de las vocales del portugués producidas por aprendices de portugués como lengua extranjera. 
(2) Comparar la estructura de los formantes F1 y F2 de las vocales del portugués producidas por hablantes nativos (Gigliotti, 1994) y las producidas por aprendices de portugués.

(3) Efectuar, con F1 y F2, triángulos acústicos de la producción de los aprendices y de hablantes nativos.

(4) Comparar los triángulos acústicos de las dos producciones.

(5) Señalar la posible utilidad de un análisis acústico en la enseñanza del portugués como lengua extranjera.

\section{Metodología}

En relación a la metodología que guió esta investigación, se seleccionaron doce aprendices de portugués con las siguientes características:

Hablantes nativos de español venezolano.

Sexo masculino.

Adultos (entre 25-30 años) .

Inscriptos el nivel II de portugués como lengua extranjera en Fundaidiomas (Extensión de la Universidad de Los Andes. Mérida-Venezuela) El curso tiene un total de cuatro niveles.

Asistencia regular a clases.

Interesados en actividades de lectura e interpretación de diálogos.

Sin patología de lenguaje.

Con estudios universitarios concluidos o por concluir.

\begin{tabular}{|l|c|c|c|}
\hline Identificación & Edad & Procedencia & Profesión \\
\hline Inf. 1 & 27 & Mérida & Economista \\
\hline Inf. 2 & 35 & Yaracuy & Médico \\
\hline Inf. 3 & 23 & D.C & Lic. en letras \\
\hline Inf. 4 & 26 & Trujillo & Médico \\
\hline Inf. 5 & 19 & N. Esparta & $\begin{array}{c}\text { Estudiante } \\
\text { universitario }\end{array}$ \\
\hline Inf. 6 & 18 & Aragua & $\begin{array}{c}\text { Estudiante } \\
\text { universitario }\end{array}$ \\
\hline Inf. 7 & 18 & Mérida & $\begin{array}{c}\text { Estudiante } \\
\text { universitario }\end{array}$ \\
\hline Inf. 8 & 36 & Lara & \begin{tabular}{c} 
Médico \\
\hline Inf. 9
\end{tabular} \\
\hline Inf. 10 & 29 & Táchira & $\begin{array}{c}\text { Estudiante } \\
\text { universitario }\end{array}$ \\
\hline Inf. 11 & 24 & Carabobo & $\begin{array}{c}\text { Médico } \\
\text { universitario }\end{array}$ \\
\hline
\end{tabular}

Cuadro3. Informantes 


\section{Procedimiento}

Con la intención de confirmar o rechazar la hipótesis del estudio, se escogió la frase portadora "Digo... pra ele" empleada por Gigliotti (1994), en un experimento que tenía como único propósito caracterizar acústicamente las vocales del portugués brasileño producidas por hablantes nativos. La elección de esta frase, así como el sexo de los informantes, se hizo necesaria porque en este análisis se intentó una caracterización acústica de las vocales del portugués producidas por once aprendices del Nivel II, lo más cercana a la realizada por Gigliotti (1994). Por esta misma razón fueron excluidos textos espontáneos y otros tipos de corpus indicados por la sociolingüística como más apropiados.

La frase portadora "Digo... pra ele" se empleó de la siguiente forma. En el espacio en blanco, se colocaron doce logatomos (palabras monosílabas semánticamente vacías), que contenían las doce vocales el portugués. Los

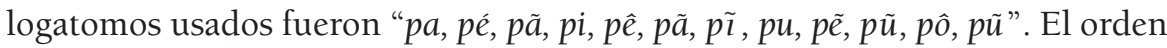
de los logatomos no obedeció a ninguna regla limitante. Se mantuvo el contexto consonante-sorda-oclusiva, por la relativa facilidad de segmentación que ofrece este contexto.

Los datos fueron registrados en un estudio de grabación acondicionado acústicamente para obtener una respuesta sonora plana, es decir, para que los sonidos grabados mantuvieran sus características físicas naturales (respuesta de frecuencia). De igual manera, estas condiciones permitieron una reproducción del sonido fiel a la emisión original y la eliminación de perturbaciones externas. Al mismo tiempo, debido a estas condiciones, se logró la manipulación electrónica de los datos. Esta manipulación, realizada por un técnico en sonido, tuvo como objetivos la organización, edición, conversión a un archivo digital, limpieza de la señal y grabación de los resultados a un CD en archivo con extensión WAV, el cual se identificó con el título de Archivo. WAV. Corpus. Data con una cuantificación de 16 bit/ 44.100 Hz.

Para la grabación, se utilizaron los siguientes instrumentos: Un micrófono con patrón polar múltiple, de respuesta plana, tipo cardioide (Newman). Este tipo de micrófono requiere alimentación Phanton, corriente de 48 voltios. Un grabador digital con un programa de grabación Macintosh, especializado en grabación y edición de sonido profesional. El programa usado fue el Pro-Tools (DigiDesgn).

Para la grabación del corpus, se procedió de la siguiente manera: a cada uno de los informantes se le entregó una hoja con las frases cinco minutos antes de comenzar la grabación. Esto con la intención de que los informan- 
tes no mecanizasen las producciones. Seguidamente, se pidió a los informantes que leyesen con voz clara, fuerte, sin repeticiones ni pausas muy prolongadas las frases, pues de esta forma se obtendría una señal continua y nítida que facilitaría el análisis acústico. Los informantes entraron en la cabina de grabación por orden de llegada. El tiempo promedio de lectura del corpus por informante fue de 31,50 seg para un total de 346,59 seg.

\begin{tabular}{|c|c|}
\hline Identificación & $\mathrm{T}$ (seg.) \\
\hline Inf. 1 & 33,5 \\
\hline Inf. 2 & 34,02 \\
\hline Inf. 3 & 32,75 \\
\hline Inf. 4 & 28,35 \\
\hline Inf. 5 & 30,58 \\
\hline Inf. 6 & 33,16 \\
\hline Inf. 7 & 29,87 \\
\hline Inf. 8 & 31,78 \\
\hline Inf. 9 & 30,78 \\
\hline Inf. 10 & 30,1 \\
\hline Inf. 11 & 31,7 \\
\hline Promedio & 31,50 \\
\hline
\end{tabular}

Cuadro.4 Tiempo promedio de grabación del corpus por informante

Para segmentar las frases, se utilizó el programa de computación Praat. La selección de las frases se realizó bajo las siguientes condiciones: Dynamic range: $30 \mathrm{~dB}$, Number of polos: 10,0 Maximum formant: $5.000 \mathrm{~Hz}$, Window length: 0,025 s, Pre-emphasis: $50.0 \mathrm{~Hz}$. La disposición del espectrograma estuvo delimitada por: Number of frequency steps: 250, View Ranger: 5000 $\mathrm{Hz}$, Window Length: 0,005 s, Maximum: 100 dB/Hz, Dynamic range: $70 \mathrm{~dB}$, Pre-emphasis: 6,0 dB/oct, Dynamic Compression: 0-1.

El Praat permitó ingresar en el archivo WAV Corpus.Data a través de la opción Read from file. Desde este archivo se seleccionaron las doce frases completas por informante. Hecha la selección, se originó una ventana que proporcionó una visión primaria en relación al tempo de emisión total de las frases. Desde esta ventana, se seleccionó la primera frase que contenía el logatomo. Esta selección generó dos ventanas: una con la onda temporal (parte superior) y otra con el espectrograma (SPG) (parte inferior). Se obtuvo de esa forma la primera frase que contenía la primera vocal.

Partiendo del espectrograma (SPG), se colocó el cursor en el inicio del segmento vocálico. La señal para determinar el inicio de la vocal fue: (1) la 
identificación de la barra de explosión de la consonante oclusiva sorda y, (2) el comienzo de las estrías que definen los pulsos glotales (porción negra en el espectrograma que muestra las vibraciones glotales). La orientación para determinar el final de la vocal fue el desaparecimiento de las vibraciones.

\section{Resultados}

Con relación a los movimientos hacia delante y hacia atrás realizados por la masa lingual al producir los fonemas vocálicos orales / a $\varepsilon$ e i $\supset$ o u / del portugués, la comparación entre los triángulos acústicos muestra que todas las vocales producidas por los aprendices se posicionan en la región posterior.

Con respecto a los movimientos hacia arriba y hacia abajo realizados por la masa lingual para producir las vocales orales / a $\varepsilon$ e i $っ$ o u / del portugués, la comparación entre los triángulos acústicos revela que todas las vocales producidas por los aprendices se elevan, esto es, se hacen más cerradas que las producidas por los hablantes nativos: la vocal $/ \varepsilon /$, definida como vocal semiabierta no presenta esta característica, puesto que como puede observarse, se eleva más que la vocal /e/, establecida como vocal semicerrada. Este mismo fenómeno se observa con las vocales /o \%/: la vocal / $/$, vocal abierta, se torna más cerrada que la vocal /o/, caracterizada como vocal semicerrada.

Se observa que, en la producción de los aprendices, no hay diferencia entre vocales semicerradas y abiertas: las vocales /o $\rho$ / casi coinciden en la misma región, mientras que las / $\varepsilon$ e/ se diferencian porque la / $\varepsilon$ / se hace más cerrada y más posterior.

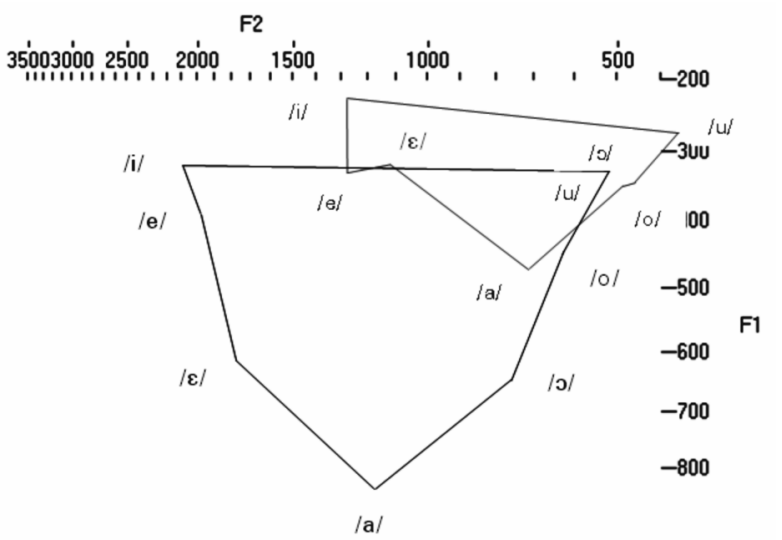

Fig. 1. Comparación de los triángulos acústicos de las vocales orales del portugués brasileño producidas por aprendices venezolanos (arriba) y las producidas por hablantes nativos (abajo). 
Con respecto a las vocales nasales / ãẽ ĩõũ / producidas por los hablantes nativos de portugués, todas las vocales nasales producidas por los once aprendices venezolanos son más altas. Las vocales / ãou /, de los aprendices, se ubican en la región posterior. Debido a esto, puede decirse que la vocal /ã / pierde la caracterización de vocal central, y se convierte, en la producción de los aprendices, en vocal nasal posterior. Observando la superposición de los triángulos acústicos, se puede afirmar que en la producción de los once aprendices venezolanos la cavidad bucal se estrecha y, como consecuencia, las vocales se cierran.

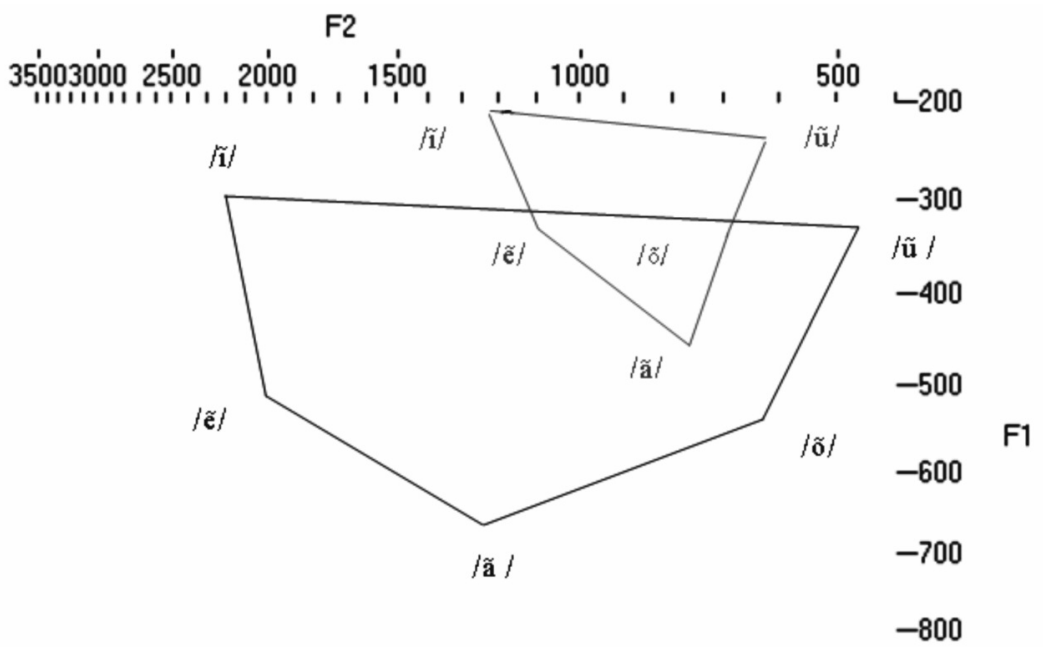

Fig. 2 Comparación de los triángulos acústicos de las vocales nasales del portugués brasileño producidas por aprendices (arriba) y las producidas por hablantes nativos (abajo).

Para finalizar, se afirma que, en relación a su cualidad acústica tanto las vocales orales como las vocales nasales del portugués brasileño, producidas por hablantes nativos, difieren de las producidas por los once aprendices venezolanos de portugués como lengua extranjera. Esto confirma la hipótesis que originó la investigación.

\section{Conclusiones}

Este trabajo plantea la estrecha interdependencia entre los movimientos de la masa lingual y las repercusiones acústicas en la producción de los fone- 
mas vocálicos. Al mismo tiempo, se pretende mostrar que tal interdependencia puede favorecer el proceso enseñanza-aprendizaje de una LE puesto que permite la realización de triángulos acústicos, que servirán como mecanismo de comparación fonética. En este caso específico, pudo comprobarse que los aprendices venezolanos de portugués como lengua extranjera no consiguieron la producción de las vocales orales semiabiertas / $\varepsilon \rho /$ del portugués brasileño. De igual modo, todas las vocales orales se realizaron en la región posterior y muy alta. El mismo efecto se observó en la producción de las vocales nasales / ã ẽ ĩ õ ũ /: muy cerradas y muy posteriores. Esta evidencia dará pistas al profesor de portugués para hablantes de español venezolano de las dificultades que tienen los aprendices para producir los fonemas vocálicos del portugués brasileño y, en consecuencia, creará estrategias de percepción y pronunciación que ayuden a los aprendices a superar dichas fallas.

\section{Recomendaciones}

Este trabajo tiene un basamento experimental que permite, desde el punto de vista pedagógico, efectuar algunas recomendaciones que beneficiarán el proceso enseñanza-aprendizaje del portugués para hispanohablantes. Entre las más significativas, se proponen:

1. Contemplar el componente fonético fonológico de la competencia comunicativa, ya que es por medio de este conocimiento que los aprendices se apropian de los rasgos distintivos de la lengua que quieren aprender; en otras palabras, debe alertarse a los aprendices sobre las realidades fonético-fonológicas de la nueva lengua. Este saber lingüístico permitirá crear significantes que facilitarán una mejor comunicación. Sólo así, la fonética y la fonología serán herramientas valiosísimas al servicio de la tan anhelada competencia comunicativa entre los aprendices de lenguas extranjeras o segundas lenguas.

2. Destacar la importancia del análisis acústico, tanto de la lengua que se enseña como de la lengua materna de los aprendices, puesto que los resultados de ese análisis aportarán los datos necesarios para la elaboración de los triángulos acústicos de ambas lenguas. Los triángulos acústicos serán la conexión que permitirá conjeturar sobre las diferencias y semejanzas entre los dos sistemas lingüísticos. Al mismo tiempo, en base a los resultados del análisis acústico, podrán proponerse estrategias que servirán de apoyo a los aprendices en el camino hacia la lengua meta.

3. Desarrollar mecanismos de percepción y producción de los nuevos sonidos con la finalidad de ir creando la conciencia fonológica a la que alude Cagliari (1978). 
4. Alertar a los aprendices sobre los sonidos de la lengua meta que se asemejan a los de su lengua materna, pues aunque se perciban parecidos no necesariamente lo son.

5. Incluir en las tareas diarias ejercicios de pronunciación, entendida como una destreza que el aprendiz debe desarrollar y dominar cunado aprende una lengua extranjera o una segunda lengua.

6. Enfatizar el uso de pares mínimos con los que el alumno logre agudizar su oído a las nuevas realidades fonéticas que la lengua meta le ofrece.

\section{Referencias bibliográficas}

Brown, D. (2000): Principles of language learning and teaching. London, Longman. Buarque, A. (1999): Novo Aurélio Século XXI. Rio de Janeiro, Nova Fronteira.

Cabrera, P. (2001): Claves didácticas para la enseñanza de lengua extranjera. Málaga, Aljibe.

Cagliari, L. (1978): A fonética e o ensino de língua estrangeira (Trabajo sin publicar). São Paulo, UNICAMP.

Canale, M. (1983): "From communicative competence to communicative language pedagogy". En J. C. Richards y R. W. Schmidt (eds.): Language and communication. London, Longman.

Corder, P. (1992): Introducción a la lingüistica aplicada. México, Limusa.

Cristófaro, T. (2001): Fonética e fonologia do português. São Paulo, Contexto.

Dubois, J. (1973): Diccionario de lingüistica. Madrid, Alianza.

Ferreira, I. (1997a): "Interface português/espanhol". En J. Almeida (comp.): Parâmetros atuais para o ensino de português lingua estrangeira. São Paulo, Pontes, págs. 141-151.

Ferreira, I. (1995b): "A Interlíngua do falante de espanhol e o papel do professor: aceitação tácita ou ajuda para superá-la”. En J. Almeida (comp.): Português para estrangeiros interface com o espanhol. São Paulo, Pontes, págs. 39-48.

Gass, S. (1996): "Second language acquisition and linguistic theory: the role of language transfer". En William C. Ritchie y T. Bhatia (eds.): Handbook of second language acquisition. Academic Press, California, págs. 317-341.

Gigliotti, E. (1994): "Para uma caracterização fonético-acústica da nasalidade no português do Brasil": http://libdigi.unicamp.br/document/?code=vtls00008 $2329 \& \mathrm{fd}=\mathrm{y}(10-08-2010)$

Hammerly, H. (1982): Synthesis in second language teaching. USA, Second language publications.

Hymes, D. (1972): "On communicative competence". En J. B. Price y J. Holmes (eds.): Sociolinguistics. Baltimore, Penguin Books.

Jakobson, R. (1973): Fundamentos del lenguaje. Madrid, Ayuso.

Lado, R. (1973): Lingüistica contrastiva, lengua y cultura. Madrid, Alcalá. 
Llisterri, J. (2003). "La enseñanza de la pronunciación", http://liceu.uab.es/ joaquim/applied_linguistics/IC_Paris_03/Guion_bibliografia.pdf (10-08-2010).

Martinet, J. (1972): La lingüística. Guía alfabética. Barcelona, Anagrama.

Muñoz, C. (2000): Segundas lenguas. Adquisición en aula. Barcelona, Ariel.

Quilis, A. (1988): Fonética acústica de la lengua española. Madrid, Gredos

Santos, P. (1998): Ensino e pesquisa em português para estrangeiros. Brasilia, Universidad de Brasilia.

Savignon, S. (1971): "A study of the effect of training in communicative skills as part of a beginning college", http://myais.fsktm.um.edu.my/3459/ - 38k (10-08-2010).

Selinker, L. (1972): “Interlanguage”, IRAL, 10, págs. 131-210.

Trubetzkoy, N. (1973): Principios de fonología. Madrid, Cincel.

Valdman, A. (1975): "On the specification of performance objectives in individual foreign-language instruction", The Modern Language Journal, 59, pág. 353-360.

Van Ek, J. (1986): "Objectives for Foreign Language Learning",

http://www.cceyaounde.org/paginas/ele/diccionarioELE_cvc/dic... - 202k - (10-08-2010). 